July 1992

HLRZ Jülich 92-47

Bern BUTP-92/34

\title{
The confined-deconfined Interface Tension and the Spectrum of the Transfer Matrix
}

\author{
B. Grossmann ${ }^{1}$, M. L. Laursen ${ }^{1}$, T. Trappenberg ${ }^{1,2, *}$ \\ and U.-J. Wiese ${ }^{3, \#}$ \\ ${ }^{1}$ HLRZ c/o KFA Jülich, P.O.Box 1913, 5170 Jülich, Germany \\ ${ }^{2}$ Institute of Theoretical Physics E, RWTH Aachen, \\ Sommerfeldstr., 5100 Aachen, Germany \\ 3 Universität Bern, Sidlerstrasse 5, 3012 Bern, Switzerland
}

\begin{abstract}
The reduced tension $\sigma_{c d}$ of the interface between the confined and the deconfined phase of $S U(3)$ pure gauge theory is related to the finite size effects of the first transfer matrix eigenvalues. A lattice simulation of the transfer matrix spectrum at the critical temperature $T_{c}=1 / L_{t}$ yields $\sigma_{c d}=0.139(4) T_{c}^{2}$ for $L_{t}=2$. We found numerical evidence that the deconfined-deconfined domain walls are completely wet by the confined phase, and that the confined-deconfined interfaces are rough.
\end{abstract}

*Supported by the Deutsche Forschungsgemeinschaft.

\#Supported by the Schweizer Nationalfond. 


\section{Introduction}

At high temperatures $Q C D$ does not confine color and chiral symmetry is not spontaneously broken. In the early universe, at about $10^{-3}$ seconds after the big bang, quarks and gluons have passed a transition to the low temperature confined phase in which chiral symmetry is spontaneously broken. Depending on the quark masses the transition may be a first or second order phase transition or just a cross over. At a first order phase transition the nucleation of bubbles of the confined phase from the supercooled high temperature quarkgluon plasma produces spatial inhomogeneities in the baryon density. If such inhomogeneities are large enough, they may influence the primordial nucleosynthesis of light elements which is completed within the first minute after the big bang. Small fluctuations, on the other hand, are washed out and the baryon density at the time of nucleosynthesis becomes homogeneous. The size of the inhomogeneities is governed by the value of the interface tension between the high and the low temperature phase. It is convenient to introduce the reduced interface tension

$$
\sigma_{c d}=\frac{F}{A T}
$$

where $F$ is the interface free energy, $A$ is the interface area and $T$ is the temperature. Computing $\sigma_{c d}$ is a nonperturbative problem which is numerically very difficult when quarks are present. Light quarks have a tendency to weaken the phase transition. To obtain an upper limit for the interface tension it is therefore sufficient to neglect quarks and to restrict oneself to a pure $S U(3)$ gauge theory of only gluons. Then, as numerical lattice simulations have shown [1], the phase transition is first order. The value of the reduced interface tension has been determined numerically at the critical temperature $T_{c}=1 / L_{t}$ where $L_{t}$ is the lattice extent in the euclidean time direction. For $L_{t}=2$ the Boston group obtained $\sigma_{c d}=0.12(2) T_{c}^{2}$ [2] while the Helsinki group quotes $\sigma_{c d}=0.08(2) T_{c}^{2}$ [3]. In this paper, using a completely different method, we obtain $\sigma_{c d}=0.139(4) T_{c}^{2}$. Closer to the continuum limit for $L_{t}=4$ the Boston group quotes $\sigma_{c d}=0.027(4) T_{c}^{2}$ [4]. Taking this value as an estimate of the interface tension in real QCD with quarks the typical scale of inhomogeneities (e.g. the distance between nucleation centers) is at most a few centimeters [5]. The proton diffusion length, on the other hand, is much larger (about $50 \mathrm{~cm}$ ) such that the fluctuations are washed out before primordial nucleosynthesis takes place.

In previous numerical studies of the interface tension coexistence of the confined and the deconfined phase was enforced by keeping different parts of the lattice at different temperatures or by applying an external field. This breaks translation invariance and pins the interface at a certain position. The properties of a pinned interface will in general be different from the ones of the free interface one is interested in. To extract the interface tension one should first perform the infinite volume limit and then turn off the temperature gradient or the external field. In practice this is difficult because in a numerical simulation the lattice size is necessarily limited. This causes finite size effects which must be understood before one can extrapolate results reliably to the infinite volume limit. In this work we use a different strategy. We make use of the fact that the finite volume alone supports coexisting bulk phases when a cylindrical geometry is used. The lattices we use have two rather short $x$ and $y$-directions and one much longer $z$-direction. Then, without artificial temperature gradients or external fields, several bulk phases, aligned along the $z$-direction, coexist with each other, separated by confined-deconfined interfaces spanned in the short $x$ - and $y$-directions. The presence of the interfaces influences the spectrum of the transfer matrix in the long $z$-direction. In particular, the lowest energy levels show a characteristic dependence on the interface area and on the reduced interface tension $\sigma_{c d}$. We will use this finite size effect to 
extract the value of $\sigma_{c d}$. This method has been applied before to the four-dimensional [6] and to the three-dimensional Ising model [7]. It has the advantages that no pinning of the interfaces is necessary and that finite size effects are well understood such that the results of numerical simulations can be extrapolated reliably to the infinite volume limit.

For the pure glue theory, however, additional phenomena arise. Since in the high temperature phase the $\mathbf{Z}(3)$ center symmetry of the nonabelian gauge group is spontaneously broken there are actually three deconfined phases. At temperatures above $T_{c}$ these phases coexist. They are separated by deconfined-deconfined domain walls with another reduced interface tension $\sigma_{d d}(T)$ which depends on the temperature. At very high temperatures $\left(T \gg \Lambda_{Q C D}\right)$ the reduced interface tension has been computed semiclassically [8]

$$
\sigma_{d d}(T)=\frac{8 \pi^{2}}{9 g^{2}} T^{2},
$$

where $g$ is the gauge coupling renormalized at the scale $T$. Frei and Patkós [9] have suggested that

$$
\sigma_{d d}\left(T_{c}\right)=2 \sigma_{c d},
$$

i.e. at the transition temperature a deconfined-deconfined domain wall costs the same free energy as two confined-deconfined interfaces. In this situation - called complete wetting the phase transition proceeds via domain wall splitting. Already slightly above the transition temperature the deconfined-deconfined domain walls split into two confined-deconfined interfaces by creating a complete wetting layer of confined phase between two bulk deconfined phases [12]. Complete wetting is a critical phenomenon of interfaces which arises although the bulk phase transition is first order and not universal. The critical exponents of complete wetting were determined in ref. [13]. In particular, the thickness of the confined wetting layer grows to a macroscopic size as

$$
z_{0} \propto-\log \left(T-T_{c}\right),
$$

and the expectation value of the Polyakov loop at the interface vanishes as

$$
\Phi_{1}(0) \propto-\sqrt{T-T_{c}} .
$$

Numerical evidence for complete wetting has been reported for the $S U(3)$ gauge theory [3, 10] and for the three state Potts model [11]. Complete wetting causes characteristic finite volume effects in the spectrum of the transfer matrix [13]. They can be used to identify complete wetting in a numerical simulation and to determine the value of the reduced interface tension $\sigma_{c d}$. When wetting is incomplete, i.e. when $\sigma_{d d}\left(T_{c}\right)<2 \sigma_{c d}$, the transfer matrix spectrum is different but again allows to determine the values of the interface tensions [13]. Wetting is a phenomenon which arises only in the pure glue system. In the real world with quarks the $\mathbf{Z}(3)$ center symmetry is explicitly broken. Therefore, two of the three deconfined phases are only metastable. In the early universe the metastable phases have converted into the stable one already $10^{-14}$ seconds after the big bang [14]. Consequently, deconfined-deconfined interfaces did not survive until the QCD phase transition and wetting could not arise. To avoid complications due to wetting in numerical simulations of the pure glue system one may choose $C$-periodic boundary conditions [15]. Just like quarks they break the $\mathbf{Z}(3)$ center symmetry explicitly and eliminate two of the three deconfined phases [16]. Therefore, in a $C$-periodic volume the confined phase coexists with only one deconfined phase such that deconfined-deconfined domain walls are absent.

In order to apply the finite size formulae derived in ref. [13] one has to be at the finite volume critical temperature, i.e. at the temperature where the free energies of the confined 
and the deconfined phases are equal. In practice this temperature is not known with high enough precision. Therefore, in this paper we generalize the finite size formulae to temperatures slightly off $T_{c}$ using a dilute interface approximation. We distinguish the cases of complete and incomplete wetting and we also consider systems with $C$-periodic boundary conditions. For a wide class of models similar formulae have been derived rigorously by Borgs and Imbrie $18 \|$ for rigid interfaces. The case of the Potts model has been worked out in great detail by Borgs [19]. Wang and DeTar [20] applied similar formulae to results of numerical simulations of the three state Potts model.

In three dimensions interfaces living on a lattice have a roughening transition [21]. For $S U(3)$ pure lattice gauge theory with a fixed number of lattice points in the euclidean time direction we expect that the deconfined-deconfined interfaces become rigid deep in the deconfined phase, i.e. when the Wilson coupling $\beta$ becomes large. A rigid interface is more or less flat with only small steplike excitations following the lattice structure. Rigid interfaces are therefore lattice artifacts. (Of course, in condensed matter systems the lattice often has physical reality. Then rigid interfaces can exist in nature.) Below the roughening transition, which we believe to be at $\beta$-values well above the deconfinement phase transition, the interfaces fluctuate more freely. In particular, there exist soft modes - the so-called capillary waves - which dominate the interface fluctuations [21]. We expect that confineddeconfined interfaces are always rough. Of course, at any given physical temperature also the deconfined-deconfined interfaces should become rough and hence forget about the lattice structure in the continuum limit. The continuum limit is also taken at large $\beta$, but now the temperature remains fixed in physical units of e.g. the inverse bulk correlation length. The finite size formulae we are using to extract the confined-deconfined reduced interface tension are valid both for rough and for rigid interfaces. Still, a comparison with the results of Borgs and Imbrie [18 allows to distinguish between the two possibilities.

The paper is organized as follows. In section 2 the $\mathbf{Z}(3)$-symmetry and the behavior of the Polyakov loop are discussed in periodic and in $C$-periodic volumes. In section 3 the finite size effects of the spectrum of the transfer matrix are derived for complete and for incomplete wetting as well as in the case of $C$-periodic boundary conditions. Section 4 contains the results of our numerical simulations on the subject of wetting and the determination of the reduced confined-deconfined interface tension $\sigma_{c d}$. Finally, in section 5 we summarize our results and we draw some conclusions. In the appendix we give some details of the formulae used in section 3 to derive the spectrum in the vicinity of the phase transition.

\section{Polyakov loops in periodic and in $C$-periodic volumes}

Let us consider an $S U(3)$ pure gauge theory on an $L_{x} \times L_{y} \times L_{z} \times L_{t}$ lattice corresponding to a temperature $T=1 / L_{t}$. The link variables $U_{\mu}(\vec{x}, t) \in S U(3)$ are periodic in the euclidean time direction

$$
U_{\mu}\left(\vec{x}, t+L_{t}\right)=U_{\mu}(\vec{x}, t) .
$$

The order parameter for the deconfinement phase transition is the Polyakov loop

$$
\Phi(\vec{x})=\operatorname{Tr} \prod_{t=1}^{L_{t}} U_{4}(\vec{x}, t) .
$$

$\Phi(\vec{x})=\Phi_{1}(\vec{x})+i \Phi_{2}(\vec{x})$ is a complex scalar field in three dimensions. The Polyakov loop is invariant under periodic gauge transformations $g(\vec{x}, t) \in S U(3)$. However, under transformations

$$
g\left(\vec{x}, t+L_{t}\right)=g(\vec{x}, t) z,
$$


which are periodic only up to a center element $z \in \mathbb{Z}(3)=\{\exp (2 \pi i n / 3), n=1,2,3\}$ it transforms as

$$
\Phi(\vec{x})^{\prime}=\Phi(\vec{x}) z
$$

while the (Wilson) action remains invariant. Hence, the Polyakov loop indicates if the $\mathbf{Z}(3)$ center symmetry is spontaneously broken. This is the case at high temperatures. Then color is deconfined and an external static quark has a finite free energy $F$ such that $\langle\Phi\rangle \propto$ $\exp (-F / T) \neq 0$. At low temperatures, because of confinement, the free energy diverges such that $\langle\Phi\rangle=0$ and the $\mathbf{Z}(3)$-symmetry is unbroken. The two phases are separated by a first order phase transition [1]. At the transition temperature the confined phase coexists with three degenerate deconfined phases which are distinguished by the expectation value of the Polyakov loop. There is one phase with a real value $\langle\Phi\rangle=\Phi^{(1)}=\left(\Phi_{0}, 0\right)$. The other two deconfined phases are $\mathbb{Z}(3)$ copies of it with $\langle\Phi\rangle=\Phi^{(2)}=\left(-\frac{1}{2} \Phi_{0}, \frac{\sqrt{3}}{2} \Phi_{0}\right)$ and with $\langle\Phi\rangle=\Phi^{(3)}=\left(-\frac{1}{2} \Phi_{0},-\frac{\sqrt{3}}{2} \Phi_{0}\right)$.

In the numerical simulations we use lattices with a cylindrical geometry, i.e. with two short $x$ - and $y$-directions and one much longer $z$-direction. Then close to the critical temperature typical configurations consist of several coexisting bulk phases, aligned along the $z$ direction, and separated by interfaces which are spanned in the transverse $x$ - and $y$-directions. In all calculations we use $C$-periodic boundary conditions [15] in the $z$-direction, i.e.

$$
U_{\mu}\left(\vec{x}+L_{z} \vec{e}_{z}, t\right)={ }^{C} U_{\mu}(\vec{x}, t)=U_{\mu}^{*}(\vec{x}, t) .
$$

A $C$-periodic field is replaced by its charge conjugate when it is shifted over the boundary. Eq. 2.5) implies $C$-periodic boundary conditions also for the Polyakov loop, i.e.

$$
\Phi\left(\vec{x}+L_{z} \vec{e}_{z}\right)=\Phi^{*}(\vec{x}) .
$$

This allows the existence of any number of deconfined-deconfined interfaces, while with periodic boundary conditions the number of interfaces is necessarily even. One can also use $C$-periodic boundary conditions in the $x$ - and $y$-directions, i.e.

$$
U_{\mu}\left(\vec{x}+L_{x} \vec{e}_{x}, t\right)=U_{\mu}\left(\vec{x}+L_{y} \vec{e}_{y}, t\right)=U_{\mu}^{*}(\vec{x}, t) .
$$

The $C$-periodic action is invariant under $C$-periodic gauge transformations

$$
g\left(\vec{x}+L_{i} \vec{e}_{i}, t\right)=g^{*}(\vec{x}, t)
$$

Considering again a transformation which is up to a center element periodic in the euclidean time direction one finds

$$
g^{*}(\vec{x}, t)=g^{*}\left(\vec{x}, t+L_{t}\right) z=g\left(\vec{x}+L_{i} \vec{e}_{i}, t+L_{t}\right) z=g\left(\vec{x}+L_{i} \vec{e}_{i}, t\right) z^{2}=g^{*}(\vec{x}, t) z^{2} .
$$

Consistency requires $z^{2}=1$ and hence $z=1$ (because $z \in \mathbf{Z}(3)$ ). The transformations of eq.(2.3) are inconsistent with $C$-periodic boundary conditions unless $z=1$. Hence, in a $C$-periodic volume the $\mathbf{Z}(3)$-symmetry is explicitly broken by the boundary conditions. With $C$-periodic boundary conditions in the $x$ - and $y$-directions the Polyakov loop obeys

$$
\Phi\left(\vec{x}+L_{x} \vec{e}_{x}\right)=\Phi\left(\vec{x}+L_{y} \vec{e}_{y}\right)=\Phi^{*}(\vec{x}) .
$$

In particular, the two deconfined phases $\Phi^{(2)}$ and $\Phi^{(3)}$ with non-real expectation values of the Polyakov loop are excluded by the boundary conditions. Hence, in a $C$-periodic volume the confined phase coexists only with the deconfined phase $\Phi^{(1)}$ [16. This excludes complications 
due to wetting because deconfined-deconfined domain walls cannot even exist. On the other hand, when periodic boundary conditions are used in the short $x$ - and $y$-directions all three deconfined phases coexist with the confined phase. In this situation one can study questions of wetting.

In the simulations we measure correlation functions $\left\langle\mathcal{O}_{i}(0) \mathcal{O}_{i}(z)\right\rangle$ in the long $z$-direction. The operators we use are the real and imaginary parts as well as the absolute value of the Polyakov loop

$$
\begin{aligned}
\mathcal{O}_{1}(z) & =\sum_{x, y} \Phi_{1}(x, y, z) \\
\mathcal{O}_{2}(z) & =\sum_{x, y} \Phi_{2}(x, y, z) \\
\mathcal{O}_{3}(z) & =\sum_{x, y}|\Phi(x, y, z)|^{2}
\end{aligned}
$$

The operators $\mathcal{O}_{1}$ and $\mathcal{O}_{3}$ are even under charge conjugation and their correlation functions are therefore periodic. The operator $\mathcal{O}_{2}$, on the other hand, is $C$-odd and its correlation function is hence anti-periodic. A $C$-odd correlation function is easier to measure in a numerical simulation because it gets contributions from single deconfined-deconfined interfaces while a nontrivial contribution to a $C$-even correlation function requires at least two interfaces and is therefore more suppressed.

\section{The interface tension and the spectrum of the trans- fer matrix}

The transfer matrix $\mathcal{T}(z)$ describes the evolution of the gluonic system over a distance $z$ in the spatial $z$-direction. In field representation it can be defined as the transition amplitude between an initial Polyakov loop distribution $\Phi^{(i)}(x, y)$ and a final distribution $\Phi^{(j)}(x, y)$, i.e. as a path integral

$$
\mathcal{T}_{i j}(z)=\int \mathcal{D} U \exp (-S[U])
$$

where $S[U]$ is the gluon action. The integration is restricted to field configurations in the interval $[0, z]$ for which $\Phi(x, y, 0)=\Phi^{(i)}(x, y)$ and $\Phi(x, y, z)=\Phi^{(j)}(x, y)$. The transfer matrix can be written as

$$
\mathcal{T}(z)=\exp (-H z)
$$

The spectrum of $H$ shows characteristic finite size effects which allow to distinguish between complete and incomplete wetting and which can be used to determine the value of the reduced confined-deconfined interface tension.

For simplicity let us start with a system with $C$-periodic boundary conditions in the short $x$ - and $y$-directions. Then only the deconfined phase $\Phi^{(1)}$ coexists with the confined phase. In the subspace of these two phases the transfer matrix takes the form

$$
t(z)=\left(\begin{array}{ll}
t_{d d}(z) & t_{c d}(z) \\
t_{c d}(z) & t_{c c}(z)
\end{array}\right)
$$

where $t_{d d}(z)$ is the transition amplitude from the deconfined phase back to itself, $t_{c d}(z)$ describes transitions from the confined phase to the deconfined phase (or vice versa) and 
$t_{c c}(z)$ is the amplitude for transitions from the confined phase back to the confined phase. The eigenvalues of the transfer matrix are

$$
\begin{aligned}
& t_{0}(z)=\frac{1}{2}\left[t_{d d}(z)+t_{c c}(z)+\sqrt{\left(t_{d d}(z)-t_{c c}(z)\right)^{2}+4 t_{c d}(z)^{2}}\right], \\
& t_{1}(z)=\frac{1}{2}\left[t_{d d}(z)+t_{c c}(z)-\sqrt{\left(t_{d d}(z)-t_{c c}(z)\right)^{2}+4 t_{c d}(z)^{2}}\right] .
\end{aligned}
$$

In ref. 13] the amplitudes $t_{d d}(z), t_{c d}(z), t_{c c}(z)$ were computed in the dilute interface approximation assuming that the free energies of all phases are equal, i.e. that one is exactly at the finite volume critical temperature. In practice, this temperature is not known with high enough precision. Therefore, to interpret the numerical data correctly, it is necessary to understand the transfer matrix spectrum also slightly off $T_{c}$. This requires to take the free energy difference between the confined and the deconfined phases into account. In the dilute interface calculation we perform a path integral over configurations which consist of several bulk phases, aligned along the $z$-direction, and separated by interfaces spanned in the $x$-y-plane. A block of confined phase of thickness $z_{0}$ costs the free energy $F=f_{c} A z_{0}$, where $f_{c}$ is the free energy density of the confined phase and $A=L_{x} L_{y}$ is the area in the transverse directions. Hence, such a block of confined phase is weighted with a Boltzmann factor

$$
\exp (-F / T)=\exp \left(-f_{c} A z_{0} / T\right)
$$

Similarly, a block of deconfined phase gets a factor

$$
\exp (-F / T)=\exp \left(-f_{d} A z_{0} / T\right)
$$

We can take out an overall factor $\exp \left(-\frac{1}{2}\left(f_{c}+f_{d}\right) A z_{0} / T\right)$ without changing the energy differences. Following ref. [19] we introduce the variable

$$
x=\frac{1}{2}\left(f_{c}-f_{d}\right) A / T .
$$

Then we write a factor $\exp \left(-x z_{0}\right)$ for the confined phase and a factor $\exp \left(x z_{0}\right)$ for the deconfined phase. The bulk phases are separated by interfaces which cost a free energy $F=\sigma_{c d} A T$, where $\sigma_{c d}$ is the reduced confined-deconfined interface tension. An interface gets the Boltzmann factor

$$
\exp (-F / T)=\delta \exp \left(-\sigma_{c d} A\right)
$$

where the pre-exponential factor $\delta$ arises due to capillary wave fluctuations of the interface (which of course also influence the value of $\sigma_{c d}$ ). In general, one would expect that $\delta$ also depends on the interface area $A$. However, in three dimensions the leading contribution to $\delta$ is $A$-independent [17. We treat $\delta$ as an unknown constant. In ref. 18 the pre-exponential factor was computed for rigid interfaces as $\delta=1$. We expect that our interfaces are rough and that these results do not apply here.

Let us write down the leading contributions to the amplitude $t_{d d}(z)$;

$$
\begin{aligned}
& t_{d d}(z)=\exp (x z)+\int_{0}^{z} d z_{0} \int_{z_{0}}^{z} d z_{0}^{\prime} \\
& \exp \left(x z_{0}\right) \delta \exp \left(-\sigma_{c d} A\right) \exp \left(-x\left(z_{0}^{\prime}-z_{0}\right)\right) \delta \exp \left(-\sigma_{c d} A\right) \exp \left(x\left(z-z_{0}^{\prime}\right)\right)+\ldots .
\end{aligned}
$$

The first term describes a block of deconfined phase of thickness $z$ filling the whole interval. The second term is the two-interface contribution. One integrates over the positions $z_{0}$ and 
$z_{0}^{\prime}$ of the two interfaces. The first factor in the integrand stands for a deconfined bulk phase of thickness $z_{0}$. The next factor describes the interface located at $z_{0}$ followed by a block of confined bulk phase of thickness $z_{0}^{\prime}-z_{0}$. Next comes the factor for the interface located at $z_{0}^{\prime}$ and finally a deconfined bulk phase of thickness $z-z_{0}^{\prime}$. Graphically one may write eq.(3.9) as

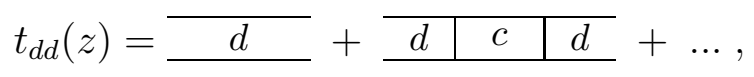

and in complete analogy one constructs the expression

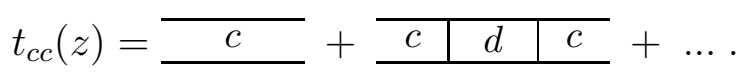

In fact one gets $t_{c c}(z)$ from $t_{d d}(z)$ by replacing $x$ by $-x$. Similarly one writes

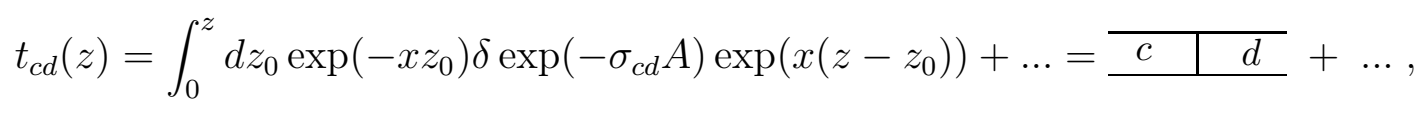

which is the one-interface contribution to the transition amplitude between the deconfined and the confined phase. It is straightforward, although somewhat tedious, to work out the multi-interface contributions and we refer the reader to the appendix for details. Using the variable $\delta^{\prime}=\delta \exp \left(-\sigma_{c d} A\right)$ the different terms are

$$
\begin{aligned}
& t_{d d}(z)=\cosh \left(z \sqrt{x^{2}+\delta^{\prime 2}}\right)+\frac{x}{\sqrt{x^{2}+{\delta^{\prime}}^{2}}} \sinh \left(z \sqrt{x^{2}+\delta^{\prime 2}}\right), \\
& t_{c c}(z)=\cosh \left(z \sqrt{x^{2}+\delta^{\prime 2}}\right)-\frac{x}{\sqrt{x^{2}+\delta^{\prime 2}}} \sinh \left(z \sqrt{x^{2}+\delta^{\prime 2}}\right), \\
& t_{c d}(z)=\frac{\delta^{\prime}}{\sqrt{x^{2}+\delta^{\prime 2}}} \sinh \left(z \sqrt{x^{2}+\delta^{\prime 2}}\right)
\end{aligned}
$$

leading to the eigenvalues

$$
\begin{aligned}
& t_{0}(z)=\exp \left(z \sqrt{x^{2}+\delta^{\prime 2}}\right), \\
& t_{1}(z)=\exp \left(-z \sqrt{x^{2}+\delta^{\prime 2}}\right) .
\end{aligned}
$$

According to eq.(3.2) we define $E_{i}$ by

$$
t_{i}(z)=\exp \left(-E_{i} z\right)
$$

and we find for the energy difference

$$
E_{1}-E_{0}=2 \sqrt{x^{2}+\delta^{2} \exp \left(-2 \sigma_{c d} A\right)} .
$$

At the finite volume critical temperature (i.e. for $x=0$ ) the result agrees with ref. [13]. It predicts that the energy splitting between the lowest states has a minimum at the critical temperature. At the minimum the energy splitting

$$
E_{1}-E_{0}=2 \delta \exp \left(-\sigma_{c d} A\right) \quad(\text { for } x=0)
$$

vanishes exponentially with the interface area times the reduced confined-deconfined interface tension $\sigma_{c d}$. This equation will be used to extract the value of $\sigma_{c d}$ in the numerical simulations. 
Now let us turn to the more complicated case of periodic boundary conditions in which the confined phase coexists with all three deconfined phases. As it was shown in refs. [13, 19] in the subspace of the three deconfined phases $\Phi^{(1)}, \Phi^{(2)}, \Phi^{(3)}$ and the confined phase the transfer matrix takes the form

$$
t(z)=\left(\begin{array}{cccc}
t_{d d}(z) & t_{d d^{\prime}}(z) & t_{d d^{\prime}}(z) & t_{c d}(z) \\
t_{d d^{\prime}}(z) & t_{d d}(z) & t_{d d^{\prime}}(z) & t_{c d}(z) \\
t_{d d^{\prime}}(z) & t_{d d^{\prime}}(z) & t_{d d}(z) & t_{c d}(z) \\
t_{c d}(z) & t_{c d}(z) & t_{c d}(z) & t_{c c}(z)
\end{array}\right)
$$

where $t_{d d}(z), t_{d d^{\prime}}(z), t_{c d}(z)$ and $t_{c c}(z)$ are transition amplitudes between the various phases. The transfer matrix $t(z)$ is symmetric under the permutation group $S_{3} \cong \mathbf{Z}(3) \times C$ which is only broken by the boundary conditions in the $z$-direction. This group has three irreducible representations, namely a symmetric (trivial), an antisymmetric, and a mixed symmetric one, corresponding to the Young tableaux

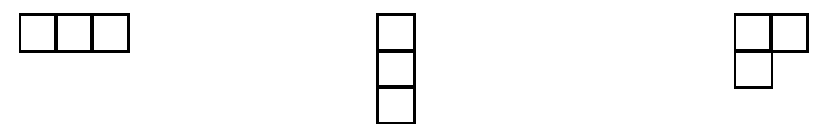

It turns out that the spectrum of the above transfer matrix splits up into a symmetric ground state with the eigenvalue $t_{0}(z)$, a corresponding excitation $t_{3}(z)$, and a twofold degenerate eigenvalue $t_{1,2}(z)$ of mixed symmetry. The eigenvectors are of the form

$$
v_{s}=\left(v_{d}, v_{d}, v_{d}, v_{c}\right)
$$

for the symmetric representation, and

$$
v_{m}=\left(v_{1}, v_{2}, v_{3}, 0\right) \text { with } \sum_{i=1}^{3} v_{i}=0
$$

for the mixed representation. For the latter we chose one basis vector $|1\rangle$ with positive $C$ parity and the other one $|2\rangle$ with negative $C$-parity. The corresponding eigenvalues of the transfer matrix are

$$
\begin{aligned}
& t_{0}(z)=\frac{1}{2}\left[t_{d d}(z)+2 t_{d d^{\prime}}(z)+t_{c c}(z)+\sqrt{\left(t_{d d}(z)+2 t_{d d^{\prime}}(z)-t_{c c}(z)\right)^{2}+12 t_{c d}(z)^{2}}\right] \\
& t_{1,2}(z)=t_{d d}(z)-t_{d d^{\prime}}(z) \\
& t_{3}(z)=\frac{1}{2}\left[t_{d d}(z)+2 t_{d d^{\prime}}(z)+t_{c c}(z)-\sqrt{\left(t_{d d}(z)+2 t_{d d^{\prime}}(z)-t_{c c}(z)\right)^{2}+12 t_{c d}(z)^{2}}\right] .
\end{aligned}
$$

Due to the $S_{3}$-symmetry the eigenvalues $t_{1}(z)$ and $t_{2}(z)$ are degenerate. We must distinguish two cases: complete and incomplete wetting.

Let us begin with the complete wetting case. Then a deconfined-deconfined domain wall consists of two widely separated confined-deconfined interfaces. Therefore, in the dilute interface approximation one must include only these interfaces, while direct deconfineddeconfined interfaces do not exist. In graphical notation the various amplitudes read

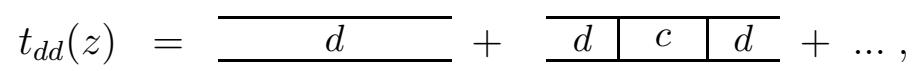

$$
\begin{aligned}
& t_{d d^{\prime}}(z)=\begin{array}{|l|l|l}
\hline d & c & d^{\prime} \\
\hline
\end{array} \\
& t_{c d}(z)=\bar{c} \mid d \quad \ldots,
\end{aligned}
$$

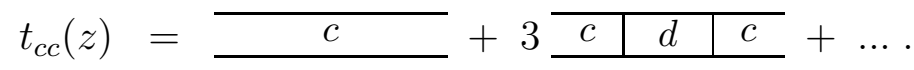


Due to complete wetting the leading contribution to the transition amplitude $t_{d d^{\prime}}(z)$ between two different deconfined phases comes from two confined-deconfined interfaces. The factor 3 in the amplitude $t_{c c}(z)$ arises because there are three possible realizations of the deconfined phase between the two blocks of confined phase. Following the appendix we now put $\delta^{\prime}=$ $\sqrt{3} \delta \exp \left(-\sigma_{c d} A\right)$. Working out the multi-interface contributions results in

$$
\begin{aligned}
\sqrt{3} t_{c d}(z) & =\frac{\delta^{\prime}}{\sqrt{x^{2}+\delta^{\prime 2}}} \sinh \left(z \sqrt{x^{2}+\delta^{\prime 2}}\right) \\
t_{c c}(z) & =\cosh \left(z \sqrt{x^{2}+\delta^{\prime 2}}\right)-\frac{x}{\sqrt{x^{2}+\delta^{\prime 2}}} \sinh \left(z \sqrt{x^{2}+\delta^{\prime 2}}\right), \\
t_{d d}(z)+2 t_{d d^{\prime}}(z) & =\cosh \left(z \sqrt{x^{2}+\delta^{\prime 2}}\right)+\frac{x}{\sqrt{x^{2}+\delta^{\prime 2}}} \sinh \left(z \sqrt{x^{2}+\delta^{\prime 2}}\right), \\
t_{d d}(z)-t_{d d^{\prime}}(z) & =\exp (z x),
\end{aligned}
$$

where $t_{c c}(z)$ and $t_{d d}(z)+2 t_{d d^{\prime}}(z)$ are related by exchanging $x$ with $-x$. The eigenvalues are

$$
\begin{aligned}
t_{0}(z) & =\exp \left(z \sqrt{x^{2}+{\delta^{\prime}}^{2}}\right) \\
t_{1,2}(z) & =\exp (z x) \\
t_{3}(z) & =\exp \left(-z \sqrt{x^{2}+\delta^{\prime 2}}\right)
\end{aligned}
$$

resulting in the energies

$$
\begin{aligned}
& E_{1,2}-E_{0}=\sqrt{x^{2}+3 \delta^{2} \exp \left(-2 \sigma_{c d} A\right)}-x, \\
& E_{3}-E_{0}=2 \sqrt{x^{2}+3 \delta^{2} \exp \left(-2 \sigma_{c d} A\right)} .
\end{aligned}
$$

At the critical temperature (for $x=0$ ) the results are in agreement with ref. 13. To leading order in $x$ they are identical with the finite size formulae derived by Borgs [19] who neglected higher-order multi-instanton contributions. We note that the $x$-dependence disappears in the combination

$$
\left(E_{3}-E_{1,2}\right)\left(E_{1,2}-E_{0}\right)=3 \delta^{2} \exp \left(-2 \sigma_{c d} A\right) .
$$

Eq.(3.26) predicts that the energy $E_{3}-E_{0}$ has a minimum at the critical temperature $(x=0)$, at which

$$
\begin{aligned}
& E_{1,2}-E_{0}=\sqrt{3} \delta \exp \left(-\sigma_{c d} A\right) \\
& E_{3}-E_{0}=2 \sqrt{3} \delta \exp \left(-\sigma_{c d} A\right) \quad(\text { for } x=0)
\end{aligned}
$$

such that one can again read off the value of $\sigma_{c d}$. The equidistant energy splitting between the lowest levels at the critical temperature, i.e.

$$
2\left(E_{1,2}-E_{0}\right)=E_{3}-E_{0} \quad(\text { for } x=0),
$$

is characteristic for complete wetting. It disappears in the incomplete wetting case to which we now turn.

In case of incomplete wetting also direct deconfined-deconfined domain walls exist, which do not consist of two confined-deconfined interfaces. They have another reduced interface tension $\sigma_{d d}$ and they get a Boltzmann factor

$$
\exp (-F / T)=\gamma \exp \left(-\sigma_{d d} A\right) .
$$


The various amplitudes then get additional contributions

$$
\begin{aligned}
& t_{d d}(z)=\overline{\frac{d}{d}}+\begin{array}{l|l|l|}
\hline d & c & d \\
\hline
\end{array}+2 \begin{array}{l|l|l}
\hline d & d^{\prime} & d \\
\hline
\end{array}
\end{aligned}
$$

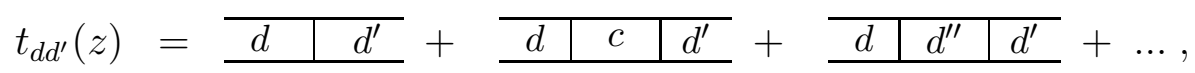

$$
\begin{aligned}
& t_{c d}(z)=\begin{array}{l|l|l|l}
\hline c & d \\
\hline
\end{array}+2 \begin{array}{c|c|c}
c & d^{\prime} & d \\
\hline
\end{array}
\end{aligned}
$$

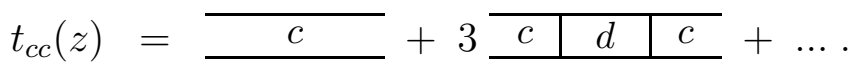

In particular, the leading contribution to the amplitude $t_{d d^{\prime}}(z)$ now comes from a direct deconfined-deconfined interface. Summing up all multi-interface contributions and using the variables $\gamma^{\prime}=2 \gamma \exp \left(-\sigma_{d d} A\right)$ and $\delta^{\prime}=\sqrt{3} \delta \exp \left(-\sigma_{c d} A\right)$ along with $x^{\prime}=x+\gamma^{\prime} / 2$ yields

$$
\begin{aligned}
\sqrt{3} t_{c d}(z) & =\frac{\delta^{\prime}}{\sqrt{x^{\prime 2}+\delta^{\prime 2}}} \sinh \left(z \sqrt{x^{\prime 2}+\delta^{\prime 2}}\right) \exp \left(z \gamma^{\prime} / 2\right) \\
t_{c c}(z) & =\left(\cosh \left(z \sqrt{x^{\prime 2}+\delta^{\prime 2}}\right)-\frac{x^{\prime}}{\sqrt{x^{\prime 2}+\delta^{\prime 2}}} \sinh \left(z \sqrt{x^{\prime 2}+\delta^{\prime 2}}\right)\right) \exp \left(z \gamma^{\prime} / 2\right) \\
t_{d d}(z)+2 t_{d d^{\prime}}(z) & =\left(\cosh \left(z \sqrt{x^{\prime 2}+\delta^{\prime 2}}\right)+\frac{x^{\prime}}{\sqrt{x^{\prime 2}+\delta^{\prime 2}}} \sinh \left(z \sqrt{x^{\prime 2}+\delta^{\prime 2}}\right)\right) \exp \left(z \gamma^{\prime} / 2\right) \\
t_{d d}(z)-t_{d d^{\prime}}(z) & =\exp (z x) \exp \left(-z \gamma^{\prime} / 2\right) .
\end{aligned}
$$

We arrive at the eigenvalues

$$
\begin{aligned}
t_{0}(z) & =\exp \left(z \sqrt{x^{\prime 2}+{\delta^{\prime}}^{2}}\right) \exp \left(z \gamma^{\prime} / 2\right) \\
t_{1,2}(z) & =\exp (z x) \exp \left(-z \gamma^{\prime} / 2\right) \\
t_{3}(z) & =\exp \left(-z \sqrt{x^{\prime 2}+\delta^{\prime 2}}\right) \exp \left(z \gamma^{\prime} / 2\right)
\end{aligned}
$$

and hence

$$
\begin{aligned}
& E_{1,2}-E_{0}=\sqrt{\left(x+\gamma \exp \left(-\sigma_{d d} A\right)\right)^{2}+3 \delta^{2} \exp \left(-2 \sigma_{c d} A\right)}-x+2 \gamma \exp \left(-\sigma_{d d} A\right), \\
& E_{3}-E_{0}=2 \sqrt{\left(x+\gamma \exp \left(-\sigma_{d d} A\right)\right)^{2}+3 \delta^{2} \exp \left(-2 \sigma_{c d} A\right)} .
\end{aligned}
$$

The combination

$$
\left(E_{3}-E_{1,2}\right)\left(E_{1,2}-E_{0}\right)=3 \delta^{2} \exp \left(-2 \sigma_{c d} A\right)-3 \gamma^{2} \exp \left(-2 \sigma_{d d} A\right)+6 x \gamma \exp \left(-\sigma_{d d} A\right)
$$

is now $x$-dependent. Furthermore, the minimum of $E_{3}-E_{0}$ no longer occurs at the critical temperature. Instead it corresponds to

$$
x=-\gamma \exp \left(-\sigma_{d d} A\right)<0,
$$

which lies in the confined phase, because $x<0$ implies that the free energy of the confined phase is smaller than the one of the deconfined phase. At the minimum one finds

$$
\begin{aligned}
& E_{1,2}-E_{0}=\sqrt{3} \delta \exp \left(-\sigma_{c d} A\right)+3 \gamma \exp \left(-\sigma_{d d} A\right), \\
& E_{3}-E_{0}=2 \sqrt{3} \delta \exp \left(-\sigma_{c d} A\right) \quad\left(\text { for } x=-\gamma \exp \left(-\sigma_{d d} A\right)\right) .
\end{aligned}
$$

As opposed to the complete wetting case the point of equidistant energy splittings between the lowest levels is now at a different position

$$
x=2 \gamma \exp \left(-\sigma_{d d} A\right)>0,
$$

which lies in the deconfined phase. It is clear that the transfer matrix spectrum is qualitatively different from the complete wetting case. 


\section{Numerical results}

We have performed numerical simulations of the $S U(3)$ pure gauge theory on lattices with $L_{t}=2$ points in the euclidean time direction close to the deconfinement phase transition. For the standard Wilson action the critical coupling is then given by $\beta=5.0933(7)$ [23]. The spatial geometry is cylindrical. We have used the sizes $L_{x} \times L_{y} \times L_{z}=4 \times 4 \times 64$, $4 \times 6 \times 64,6 \times 6 \times 64,6 \times 8 \times 96$ and $8 \times 8 \times 128$. In all cases $C$-periodic boundary conditions have been used in the long $z$-direction. In the transverse $x$ - and $y$-directions we have used both periodic and $C$-periodic boundary conditions. Of course, thermodynamics requires periodic boundary conditions for the gluon field in the euclidean time direction. We have performed measurements at several $\beta$-values very close to the critical coupling using an overrelaxed heat bath algorithm. To interpolate our results to other $\beta$-values we have used standard reweighting techniques. Per lattice we have typically performed 50000-250000 measurements.

The low energy states in the spectrum of the transfer matrix in the $z$-direction are extracted from the correlation functions of the operators $\mathcal{O}_{i}$ of eq.(2.11). Since we always use $C$-periodic boundary conditions in the $z$-direction we introduce a complete set of states $|n\rangle$ with their charge conjugate states $C|n\rangle$ and we obtain

$$
\begin{aligned}
Z\left\langle\mathcal{O}_{i}(0) \mathcal{O}_{i}(z)\right\rangle & =\sum_{n}\left\langle n\left|\mathcal{O}_{i} \exp (-H z) \mathcal{O}_{i} \exp \left(-H\left(L_{z}-z\right)\right) C\right| n\right\rangle \\
& =\sum_{m, n}\left\langle n\left|\mathcal{O}_{i}\right| m\right\rangle \exp \left(-E_{m} z\right)\left\langle m\left|\mathcal{O}_{i}\right| n\right\rangle \exp \left(-E_{n}\left(L_{z}-z\right)\right) c_{n} \\
& =\sum_{m, n} c_{n}\left|\left\langle n\left|\mathcal{O}_{i}\right| m\right\rangle\right|^{2} \exp \left(-E_{m} z\right) \exp \left(-E_{n}\left(L_{z}-z\right)\right) \\
Z & =\sum_{n}\left\langle n\left|\exp \left(-H L_{z}\right) C\right| n\right\rangle=\sum_{n} c_{n} \exp \left(-E_{n} L_{z}\right)
\end{aligned}
$$

where we have used $C|n\rangle=c_{n}|n\rangle$ for states of $C$-parity $c_{n}= \pm 1$.

For $C$-periodic boundary conditions in the $x$ - and $y$-directions only the operators $\mathcal{O}_{1}$ and $\mathcal{O}_{3}$ are relevant. Both create the state with energy $E_{1}$. Putting $E_{0}=0$ and denoting the states with energies $E_{0}$ and $E_{1}$ by $|0\rangle$ and $|1\rangle$ the transfer matrix formalism predicts

$$
\begin{aligned}
Z\left\langle\mathcal{O}_{1}(0) \mathcal{O}_{1}(z)\right\rangle & \sim\left|\left\langle 0\left|\mathcal{O}_{1}\right| 1\right\rangle\right|^{2}\left(\exp \left(-E_{1} z\right)+\exp \left(-E_{1}\left(L_{z}-z\right)\right)\right) \\
& +\left|\left\langle 0\left|\mathcal{O}_{1}\right| 0\right\rangle\right|^{2}+\left|\left\langle 1\left|\mathcal{O}_{1}\right| 1\right\rangle\right|^{2} \exp \left(-E_{1} L_{z}\right) \\
Z\left\langle\mathcal{O}_{3}(0) \mathcal{O}_{3}(z)\right\rangle & \sim\left|\left\langle 0\left|\mathcal{O}_{3}\right| 1\right\rangle\right|^{2}\left(\exp \left(-E_{1} z\right)+\exp \left(-E_{1}\left(L_{z}-z\right)\right)\right) \\
& +\left|\left\langle 0\left|\mathcal{O}_{3}\right| 0\right\rangle\right|^{2}+\left|\left\langle 1\left|\mathcal{O}_{3}\right| 1\right\rangle\right|^{2} \exp \left(-E_{1} L_{z}\right) \\
Z & \sim 1+\exp \left(-E_{1} L_{z}\right)
\end{aligned}
$$

such that

$$
\begin{aligned}
\left\langle\mathcal{O}_{1}(0) \mathcal{O}_{1}(z)\right\rangle & \sim A \cosh \left(E_{1}\left(z-\frac{1}{2} L_{z}\right)\right)+B \\
\left\langle\mathcal{O}_{3}(0) \mathcal{O}_{3}(z)\right\rangle & \sim C \cosh \left(E_{1}\left(z-\frac{1}{2} L_{z}\right)\right)+D
\end{aligned}
$$

We use these equations to determine the energy $E_{1}$ by fitting the measured correlation functions.

When periodic boundary conditions are used in the $x$ - and $y$-directions the energy splittings $E_{1,2}-E_{0}$ and $E_{3}-E_{0}$ between the excited states $|1\rangle,|2\rangle$ and $|3\rangle$ and the ground state 
$|0\rangle$ become small close to the phase transition. Applying the reduction rule

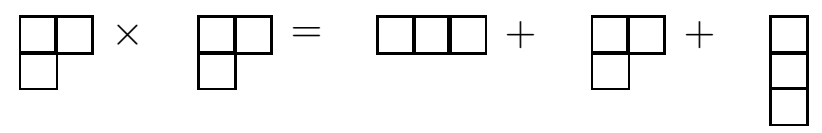

for $S_{3}$ (see 22) and again setting $E_{0}=0$ we obtain

$$
\begin{aligned}
Z\left\langle\mathcal{O}_{1}(0) \mathcal{O}_{1}(z)\right\rangle & \sim\left|\left\langle 0\left|\mathcal{O}_{1}\right| 1\right\rangle\right|^{2}\left(\exp \left(-E_{1} z\right)+\exp \left(-E_{1}\left(L_{z}-z\right)\right)\right) \\
& +\left|\left\langle 1\left|\mathcal{O}_{1}\right| 3\right\rangle\right|^{2} \exp \left(-E_{1} L_{z}\right) \\
& \times\left(\exp \left(-\left(E_{3}-E_{1}\right) z\right)+\exp \left(-\left(E_{3}-E_{1}\right)\left(L_{z}-z\right)\right)\right) \\
Z\left\langle\mathcal{O}_{2}(0) \mathcal{O}_{2}(z)\right\rangle & \sim\left|\left\langle 0\left|\mathcal{O}_{2}\right| 2\right\rangle\right|^{2}\left(\exp \left(-E_{2} z\right)-\exp \left(-E_{2}\left(L_{z}-z\right)\right)\right) \\
& -\left|\left\langle 2\left|\mathcal{O}_{2}\right| 3\right\rangle\right|^{2} \exp \left(-E_{2} L_{z}\right) \\
& \times\left(\exp \left(-\left(E_{3}-E_{2}\right) z\right)-\exp \left(-\left(E_{3}-E_{2}\right)\left(L_{z}-z\right)\right)\right) \\
Z\left\langle\mathcal{O}_{3}(0) \mathcal{O}_{3}(z)\right\rangle & \sim\left|\left\langle 0\left|\mathcal{O}_{3}\right| 3\right\rangle\right|^{2}\left(\exp \left(-E_{3} z\right)+\exp \left(-E_{3}\left(L_{z}-z\right)\right)\right) \\
& +\left|\left\langle 0\left|\mathcal{O}_{3}\right| 0\right\rangle\right|^{2}+\left|\left\langle 3\left|\mathcal{O}_{3}\right| 3\right\rangle\right|^{2} \exp \left(-E_{3} L_{z}\right) \\
Z & \sim 1+\exp \left(-E_{3} L_{z}\right)
\end{aligned}
$$

Here the additional symmetry relation $\left|\left\langle 1\left|\mathcal{O}_{i}\right| 1\right\rangle\right|^{2}=\left|\left\langle 2\left|\mathcal{O}_{i}\right| 2\right\rangle\right|^{2}$ was used. Note that the correlation function of the $C$-odd operator $\mathcal{O}_{2}$ is anti-periodic because of $C$-periodic boundary conditions in the $z$-direction. The $S_{3}$-symmetry ensures that $E_{1}=E_{2},\left|\left\langle 0\left|\mathcal{O}_{1}\right| 1\right\rangle\right|^{2}=\left|\left\langle 0\left|\mathcal{O}_{2}\right| 2\right\rangle\right|^{2}$ and $\left|\left\langle 1\left|\mathcal{O}_{1}\right| 3\right\rangle\right|^{2}=\left|\left\langle 2\left|\mathcal{O}_{2}\right| 3\right\rangle\right|^{2}$. For very large $L_{z}$ such that $E_{i} L_{z} \gg 1$ eq.(1.5) simplifies to

$$
\begin{aligned}
\left\langle\mathcal{O}_{1}(0) \mathcal{O}_{1}(z)\right\rangle & \sim A \cosh \left(E_{1}\left(z-\frac{1}{2} L_{z}\right)\right), \\
\left\langle\mathcal{O}_{2}(0) \mathcal{O}_{2}(z)\right\rangle & \sim B \sinh \left(E_{2}\left(z-\frac{1}{2} L_{z}\right)\right), \\
\left\langle\mathcal{O}_{3}(0) \mathcal{O}_{3}(z)\right\rangle & \sim C \cosh \left(E_{3}\left(z-\frac{1}{2} L_{z}\right)\right)+D .
\end{aligned}
$$

In most of our fits we have used this simpler form. However, in some cases we have checked our results using the more general expressions (4.5) and we found agreement within the error bars.

Fig.1 shows a configuration on the $8 \times 8 \times 128 \times 2$ lattice which consists of several bulk phases separated by interfaces. Two typical correlation functions are depicted in fig.2. We should mention that autocorrelation times of the correlation functions are rather long, especially at large distances. Therefore, it was always a good check if the energies $E_{1}$ and $E_{2}$ agreed within errors (as they should because of $S_{3}$-symmetry).

Fig.3a shows the energy $E_{1}$ on a $4 \times 6 \times 64 \times 2$ lattice with $C$-periodic boundary conditions in the $x$ - and $y$-directions. As expected, there is a minimum at the critical coupling. From the finite size effect of the value at the minimum we have determined the reduced confineddeconfined interface tension using eq.(3.17). The result of the fit, which is depicted in fig.4, is given by

$$
\sigma_{c d}=0.035(1)=0.140(4) T_{c}^{2} \quad(C \text {-periodic }) .
$$

The fitted value of the pre-exponential factor is $\delta=0.196(5)$ which is much smaller than the prediction $\delta=1$ for rigid interfaces [18]. We take this as an indication that the confineddeconfined interface is indeed rough. 
Figure 1: $\quad$ The $z$-dependence of $\mathcal{O}_{1}(z)=\sum_{x, y} \Phi_{1}(x, y, z)$ for a configuration on an $8 \times 8 \times 128 \times 2$ lattice with $C$-periodic boundary conditions in the spatial directions at $\beta=$ 5.0933.

Figure 2: The correlation functions $\left\langle\mathcal{O}_{1}(0) \mathcal{O}_{1}(z)\right\rangle$ and $\left\langle\mathcal{O}_{2}(0) \mathcal{O}_{2}(z)\right\rangle$ on a $4 \times 6 \times 64 \times 2$ lattice with $C$-periodic boundary condition only in the $z$-direction. The solid lines represent fits of the data to the corresponding equation (4.6). 
Figure 3: The spectra of the lowest energies in the region of the phase transition temperature. In a) the energy $E_{1}$ from simulations on a $4 \times 6 \times 64 \times 2$ lattice with $C$-periodic boundary conditions in the $x$ - and $y$-direction is shown. In b) the degenerate energy $E_{1,2}$ and the energy $E_{3}$ derived from simulations on a $6 \times 6 \times 64 \times 2$ lattice with periodic boundary conditions in the $x$ - and $y$-direction are shown. The energies are plotted as boxes at the simulation points, whereas the interpolated values are plotted as diamonds. 
Figure 4: The dependence of the energies on the cross section $A=L_{x} L_{y}$ of the cylindrical lattices. The values of $\log \left(E_{1}\right)$ are shown from simulations on lattices with $C$-periodic boundary conditions in the $x$ - and $y$-direction (diamonds) and the values of $\log \left(E_{1,2}\right)(=$ $\left.\log \left(\frac{1}{2} E_{3}\right)\right)$ are shown from simulations on lattices with periodic boundary conditions in the $x$ - and $y$-direction (squares). The solid lines represent the fits of the data to eq.(3.17) and eq. (3.28) respectively. 
Fig. 3b shows the energies $E_{1,2}$ and $E_{3}$ on a $6 \times 6 \times 64 \times 2$ lattice with periodic boundary conditions in the $x$ - and $y$-directions. As expected, the energy $E_{3}$ has a minimum which is, however, slightly shifted away from the critical $\beta$ into the deconfined phase. At the same time, the point where $2 E_{1,2}=E_{3}$ is shifted slightly into the confined phase. In case of incomplete wetting one would expect such shifts, but they would go exactly in the opposite direction. Therefore, our data favour complete wetting. Of course, then eq.(3.26) predicts that the minimum of $E_{3}$ and the point where $2 E_{1,2}=E_{3}$ should correspond to the same $\beta$-value, which is almost — but not quite - the case for our data. We believe that this effect is some sub-leading finite size effect which is not correctly reproduced by our dilute interface calculation. The same effect is visible in the data of Wang and DeTar [20] in the three-dimensional three state Potts model, which also shows complete wetting [11]. There the shift is present on smaller lattices, and it disappears on larger volumes, supporting the assumption of a sub-leading effect. Altogether, we consider fig.3b as a strong indication that the deconfined-deconfined interfaces are completely wet by the confined phase at the deconfinement phase transition. To extract the reduced confined-deconfined interface tension from our periodic lattices we have taken the value of $E_{1,2}$ at the point where $2 E_{1,2}=E_{3}$ and we have fitted its finite size behavior to eq.(3.28). The fit, which is also shown in fig.4, results in

$$
\sigma_{c d}=0.034(2)=0.136(8) T_{c}^{2} \quad \text { (periodic) } .
$$

It is reassuring (and further supports the assumption of complete wetting) that this result is in agreement with the one obtained from the $C$-periodic simulation. Also the fitted value of the pre-exponential factor, $\delta=0.202(6)$, agrees with the one obtained from the $C$-periodic data. This value again indicates that the confined-deconfined interface is rough. Furthermore, it means that the capillary waves have the same behavior in periodic and in $C$ periodic volumes. This was expected because they are small fluctuations which are insensitive to global topological characteristics of the boundary conditions. Taking the results of both boundary conditions together we state as our final answer for the reduced confined-deconfined interface tension

$$
\sigma_{c d}=0.139(4) T_{c}^{2} .
$$

We have also re-analyzed the data of Wang and DeTar [20] from our perspective. They have measured the energies $E_{1,2}$ and $E_{3}$ in the three-dimensional three state Potts model using periodic boundary conditions. As Wang and DeTar have pointed out, their results are in good agreement with complete wetting. On a $20^{2} \times 120$ lattice they find $E_{1,2}=0.048(1)$ at $\beta=0.3668$ and $E_{1,2}=0.0397(6)$ at $\beta=0.3669$ while at $\beta=0.3670$ on a $30^{2} \times 120$ lattice $E_{1,2}=0.0185(3)$. In all cases $2 E_{1,2}=E_{3}$ within the error bars. Assuming that the asymptotic formulae (3.28) are valid for these lattices we obtain

$$
\sigma_{c d}=0.0017(3) \quad(\text { Potts model) }
$$

where the error is mostly systematical. This is due to the same slight shift of the minimum of $E_{3}$ away from the point where $2 E_{1,2}=E_{3}$ which we also observed in our data. In the Potts model the shift is present only on the smaller lattice, while it disappears on the larger volume. Perhaps the result of eq.(4.10) should be taken with a grain of salt because it relies only on two different volumes. For the pre-exponential factor we find $\delta=0.042(4)$ indicating that the ordered-disordered interface is rough. The ordered-ordered reduced interface tension $\sigma_{d d}\left(T_{c}\right)$ has been determined in the Potts model by Karsch and Patkós 11. They enforced the interface by boundary conditions and computed the free energy difference due to the interface by a numerical integration. Their result

$$
\sigma_{d d}\left(T_{c}\right) \geq 0.0026(2) \quad(\text { Potts model })
$$


(translated to our notation) is consistent with eq.(4.10) because complete wetting implies $\sigma_{d d}\left(T_{c}\right)=2 \sigma_{c d}$

\section{Conclusions}

We have related the finite size effects of the spectrum of the transfer matrix to the value of the reduced confined-deconfined interface tension both for $C$-periodic and for periodic boundary conditions. In the periodic case one must distinguish between the possibilities of complete and incomplete wetting. Our numerical data strongly suggest that the deconfineddeconfined domain walls of the $S U(3)$ pure gauge theory are completely wet by the confined phase. Hence, in a hypothetical universe, which contains only gluons but no quarks (or other particles), the deconfinement phase transition would proceed via domain wall splitting and not via the usual bubble nucleation. However, the presence of quarks in our universe made wetting at the deconfinement phase transition impossible [12, 14]. Still, the reduced confined-deconfined interface tension of the pure glue system serves as an upper limit on the corresponding value in real QCD. The determination of the value $\sigma_{c d}=0.139(4) T_{c}^{2}$ required a careful analysis of finite size effects and an understanding of the wetting dynamics.

We like to mention that the transfer matrix method is a very powerful tool to extract interface properties from numerical simulations, especially because finite size effects are well understood. Certainly, in this method information on correlation functions at large distances is needed. With only local algorithms available (as it the case for gauge theories) this is not an easy task, and it requires very high statistics. An attractive alternative is the multicanonical algorithm of Berg and Neuhaus [24. We have also applied this algorithm to the pure $S U(3)$ gauge theory [25] and we have measured the interfacial free energy. It has turned out that the use of cylindrical lattices is again advantageous in order to reduce the interactions between interfaces. This work is presently in progress.

\section{Acknowledgement}

We like to thank C. Borgs and V. Privman for interesting discussions about finite size effects at first order phase transitions. One of us (T.T.) would like to thank J. Jersák for discussions. Our numerical simulations were performed on the CRAY Y-MP at the HLRZ in Jülich and on the NEC SX-3 at the University of Köln.

\section{Appendix}

In this appendix we would like to give some of the details for the formulae occurring in section 3. We shall start with the complete wetting case. Let us also assume that we have $q$ deconfined phases, such that $q=3$ for periodic and $q=1$ for $C$-periodic boundary conditions. It is convenient to define the following integrals:

$$
\begin{aligned}
I_{n}(y, \xi) & =z^{-n} \int_{\xi}^{z} d \xi_{1} \int_{\xi_{1}}^{z} d \xi_{2} \ldots \int_{\xi_{n-1}}^{z} d \xi_{n} \exp \left[y \sum_{i=1}^{n}(-1)^{i} \xi_{i}\right] \\
& =\int_{\xi}^{1} d \xi_{1} \int_{\xi_{1}}^{1} d \xi_{2} \cdots \int_{\xi_{n-1}}^{1} d \xi_{n} \exp \left[z y \sum_{i=1}^{n}(-1)^{i} \xi_{i}\right], \quad n \geq 1
\end{aligned}
$$

where $I_{n}(y, 1)=0$ and $y=2 x=\left(f_{c}-f_{d}\right) A / T$. The aim is to evaluate $I_{n}(y) \equiv I(y, 0)$, which exactly corresponds to $n$ confined-deconfined interfaces. For reasons of notational simplicity 
we rescale $z x \rightarrow x, z y \rightarrow y$, and $z \sqrt{q} \delta \exp \left(-\sigma_{c d} A\right)=z \delta^{\prime} \rightarrow \delta^{\prime}$. The different interface contributions $\sqrt{q} t_{c d}, t_{c c}$ and $t_{d d}+(q-1) t_{d d^{\prime}}$ can now be expressed in terms of $I_{n}(y)$ :

$$
\begin{aligned}
\sqrt{q} t_{c d} & =\exp (x) \sum_{n=0}^{\infty} I_{2 n+1}(y) \delta^{\prime 2 n+1}, \\
t_{c c} & =\exp (-x)\left(1+\sum_{n=1}^{\infty} I_{2 n}(y) \delta^{\prime 2 n}\right), \\
t_{d d}+(q-1) t_{d d^{\prime}} & =\exp (x)\left(1+\sum_{n=1}^{\infty} I_{2 n}(-y) \delta^{\prime 2 n}\right) .
\end{aligned}
$$

The case $n=1$ is trivial and yields

$$
\begin{aligned}
I_{1}(y, \xi) & =\frac{1}{y}(\exp (-y \xi)-\exp (-y)), \\
I_{1}(y) & =\frac{1}{y}(1-\exp (-y)) .
\end{aligned}
$$

For $n \geq 2$ we find after differentiating $I_{n}(y, \xi)$ with respect to $\xi$

$$
\frac{\partial I_{n}(y, \xi)}{\partial \xi}=-\exp (-y \xi) I_{n-1}(-y, \xi)
$$

In particular for $n=2$ :

$$
\frac{\partial I_{2}(y, \xi)}{\partial \xi}=\frac{1}{y}(1-\exp [y(1-\xi)])
$$

The integration is easy and we obtain

$$
\begin{aligned}
I_{2}(y, \xi) & =\frac{\xi-1}{y}+\frac{1}{y^{2}}(\exp [y(1-\xi)]-1), \\
I_{2}(y) & =-\frac{1}{y}+\frac{1}{y^{2}}(\exp (y)-1)=-\exp (y) \frac{\partial I_{1}(y)}{\partial y} .
\end{aligned}
$$

Repeating the steps above we arrive at

$$
\begin{aligned}
I_{2 n}(y) & =-\frac{1}{n} \exp (y) \frac{\partial I_{2 n-1}(y)}{\partial y}, \\
I_{2 n+1}(y) & =\frac{1}{n} \exp (-y) \frac{\partial I_{2 n}(y)}{\partial y}, \quad n \geq 1 .
\end{aligned}
$$

This we checked explicitly up to $n=3$. From the previous two equations follows:

$$
\begin{aligned}
& I_{2 n+1}(y)=\frac{(-1)^{n}}{n !^{2}}\left[\frac{\partial}{\partial y}+\frac{\partial^{2}}{\partial y^{2}}\right]^{n} I_{1}(y), \\
& I_{2 n+2}(y)=\frac{(-1)^{n}}{n !(n+1) !}\left[-\frac{\partial}{\partial y}+\frac{\partial^{2}}{\partial y^{2}}\right]^{n} I_{2}(y), \quad n \geq 1 .
\end{aligned}
$$

Now we can do the $\sqrt{q} t_{c d}$ term. Using the identities:

$$
\exp (x) I_{2 n+1}(2 x)=\frac{4^{n}}{n !^{2}} \sum_{i=0}^{n}\left(\begin{array}{c}
n \\
i
\end{array}\right)(-1)^{i} \frac{\partial^{2 i}}{\partial x^{2 i}}\left(\exp (x) I_{1}(2 x)\right)=\frac{1}{n !} \frac{\partial^{n}}{\left(\partial x^{2}\right)^{n}}\left(\frac{\sinh x}{x}\right)
$$


we find

$$
\sqrt{q} t_{c d}=\sum_{n=0}^{\infty} \frac{1}{n !} \frac{\partial^{n}}{\left(\partial x^{2}\right)^{n}}\left(\frac{\sinh x}{x}\right) \delta^{\prime 2 n+1}=\frac{\delta^{\prime}}{\sqrt{x^{2}+\delta^{\prime 2}}} \sinh \sqrt{x^{2}+\delta^{\prime 2}} .
$$

Now we come to $t_{c c}$. Using eqs. (A.2) and (A.7) we can write:

$$
t_{c c}=\exp (-x)-\exp (x) \frac{\partial}{\partial x} \sum_{n=1}^{\infty} \frac{1}{2 n} I_{2 n-1}(y) \delta^{2 n}
$$

Differentiating the previous equation with respect to $\delta^{\prime}$ and integrating we obtain

$$
\begin{aligned}
t_{c c} & =\exp (-x)-\exp (x) \int_{0}^{\delta^{\prime}} d \delta^{\prime} \frac{\partial}{\partial x}\left(\exp (-x) \sqrt{q} t_{c d}\right) \\
& =\cosh \sqrt{x^{2}+\delta^{\prime 2}}-\frac{x}{\sqrt{x^{2}+\delta^{\prime 2}}} \sinh \sqrt{x^{2}+\delta^{\prime 2}}
\end{aligned}
$$

Likewise we get

$$
t_{d d}+(q-1) t_{d d^{\prime}}=\cosh \sqrt{x^{2}+\delta^{\prime 2}}+\frac{x}{\sqrt{x^{2}+\delta^{\prime 2}}} \sinh \sqrt{x^{2}+\delta^{\prime 2}} .
$$

Finally we have trivially

$$
t_{d d}-t_{d d^{\prime}}=\exp (x)
$$

The case of incomplete wetting is somewhat harder. First we rescale the expression $z(q-1) \gamma \exp \left(-\sigma_{d d} A\right)=z \gamma^{\prime} \rightarrow \gamma^{\prime}$. We begin again with $\sqrt{q} t_{c d}$. To lowest non-trivial order in $\delta^{\prime}$, but to all orders in $\gamma^{\prime}$, we obtain easily

$$
\sqrt{q} t_{c d}=\exp (-x) \sum_{n=0}^{\infty} \frac{1}{n !} \frac{\partial^{n}}{\partial y^{n}} I_{1}(-y) \gamma^{\prime n}=\frac{\exp (x)}{y} \sum_{n=0}^{\infty}\left(-\frac{\gamma^{\prime}}{y}\right)^{n}\left(\sum_{i=0}^{n} \frac{(-y)^{i}}{i !}-\exp (-y)\right) .
$$

The single sum is easy

$$
\frac{1}{y} \sum_{n=0}^{\infty}\left(-\frac{\gamma^{\prime}}{y}\right)^{n}=\frac{1}{y+\gamma^{\prime}}
$$

For the double sum we first let $i \rightarrow n-i$ and then we interchange the order of the summations. This gives

$$
\frac{1}{y} \sum_{n=0}^{\infty}\left(-\frac{\gamma^{\prime}}{y}\right)^{n} \sum_{i=0}^{n} \frac{(-y)^{n-i}}{(n-i) !}=\frac{1}{y} \sum_{i=0}^{\infty}\left(-\frac{\gamma^{\prime}}{y}\right)^{i} \sum_{n=i}^{\infty} \frac{\gamma^{\prime n-i}}{(n-i) !}=\frac{\exp \left(\gamma^{\prime}\right)}{y+\gamma^{\prime}}
$$

so that with $x^{\prime}=x+\gamma^{\prime} / 2$

$$
\sqrt{q} t_{c d}=\delta^{\prime} \frac{\sinh x^{\prime}}{x^{\prime}} \exp \left(\gamma^{\prime} / 2\right)
$$

Combining eqs. (A.10) and (A.17) we conjecture that to all orders in $\delta^{\prime}$ the following holds:

$$
\sqrt{q} t_{c d}=\frac{\delta^{\prime}}{\sqrt{x^{\prime 2}+\delta^{\prime 2}}} \sinh \sqrt{x^{\prime 2}+\delta^{\prime 2}} \exp \left(\gamma^{\prime} / 2\right) .
$$

This we checked explicitly up to order $O\left(\delta^{\prime 4}\right)$. It is now easy to guess the expressions for $t_{c c}$ and $t_{d d}+(q-1) t_{d d^{\prime}}$. They are:

$$
\begin{aligned}
t_{c c} & =\left(\cosh \sqrt{x^{\prime 2}+\delta^{\prime 2}}-\frac{x^{\prime}}{\sqrt{x^{\prime 2}+\delta^{\prime 2}}} \sinh \sqrt{x^{\prime 2}+\delta^{\prime 2}}\right) \exp \left(\gamma^{\prime} / 2\right) \\
t_{d d}+(q-1) t_{d d^{\prime}} & =\left(\cosh \sqrt{x^{\prime 2}+\delta^{\prime 2}}+\frac{x^{\prime}}{\sqrt{x^{\prime 2}+\delta^{\prime 2}}} \sinh \sqrt{x^{\prime 2}+\delta^{\prime 2}}\right) \exp \left(\gamma^{\prime} / 2\right)
\end{aligned}
$$


This we also checked explicitly up to order $O\left(\delta^{\prime 4}\right)$. Notice for $\gamma^{\prime}=0$ we reproduce the results for the complete wetting case. Finally we find

$$
t_{d d}-t_{d d^{\prime}}=\exp (x) \exp \left[-\gamma^{\prime} /(q-1)\right]
$$

The formulae given in the main text are obtained by replacing

$$
x \rightarrow z x, x^{\prime} \rightarrow z x^{\prime}, \delta^{\prime} \rightarrow z \delta^{\prime}=z \sqrt{q} \delta \exp \left(-\sigma_{c d} A\right), \gamma^{\prime} \rightarrow z \gamma^{\prime}=z(q-1) \gamma \exp \left(-\sigma_{d d} A\right) .
$$

As an addendum we would like to mention the following identity:

$$
\operatorname{det}(t)=\exp \left[-\left(f_{c}+q f_{d}\right) A / T\right]
$$

which is equivalent to

$$
t_{c c}\left(t_{d d}+(q-1) t_{d d^{\prime}}\right)-\sqrt{q} t_{c d} \sqrt{q} t_{c d}=\exp \left(\gamma^{\prime}\right)
$$

\section{References}

[1] R. V. Gavai, F. Karsch and B. Petersson, Nucl. Phys. B322 (1989) 738;

M. Fukugita, M. Okawa and U. Ukawa, Phys. Rev. Lett. 63 (1989) 1768; Nucl. Phys. B337 (1990) 181;

N. A. Alves, B. A. Berg and S. Sanielevici, Phys. Rev. Lett. 64 (1990) 3107

[2] S. Huang, J. Potvin, C. Rebbi and S. Sanielevici, Phys. Rev. D42 (1990) 2864

[3] K. Kajantie, L. Kärkkäinen and K. Rummukainen, Nucl. Phys. B333 (1990) 100; Nucl. Phys. B357 (1991) 693

[4] R. Brower, S. Huang, J. Potvin and C. Rebbi, Boston preprint, BUHEP-92-3, 1992

[5] B. Banerjee and R. V. Gavai, Bombay preprint, TIFR/TH/92-24, 1992.

[6] K. Jansen, J. Jersák, I. Montvay, G. Münster, T. Trappenberg and U. Wolff, Phys. Lett. B213 (1988) 203;

K. Jansen, I. Montvay, G. Münster, T. Trappenberg and U. Wolff, Nucl. Phys. B322 (1989) 698;

K. Jansen and Y. Shen, San Diego preprint, UCSD/PTH 92-02, 1992

[7] H. Meyer-Ortmanns and T. Trappenberg, J. Stat. Phys. 58 (1990) 185;

S. Klessinger and G. Münster, Münster preprint, MS-TPI-92-13, 1992

[8] T. Bhattacharya, A. Gocksch, C. Korthals Altes and R. D. Pisarski, Phys. Rev. Lett. 66 (1991) 998

[9] Z. Frei and A. Patkós, Phys. Lett. B229 (1989) 102

[10] R. Brower, S. Huang, J. Potvin, C. Rebbi and J. Ross, Boston preprint, BUHEP-91-22, 1991

[11] F. Karsch and A. Patkós, Nucl. Phys. B350 (1991) 563

[12] U.-J. Wiese, in: "Workshop on the Dynamics of First Order Phase Transitions", HLRZ, June 1-3, 1992, ed. H. J. Herrmann and F. Karsch, World Scientific, Singapore 1992 (in press) 
[13] T. Trappenberg and U.-J. Wiese, Nucl. Phys. B372 (1992) 703

[14] J. Ignatius, K. Kajantie and K. Rummukainen, Phys. Rev. Lett. 68 (1992) 737

[15] L. Polley and U.-J. Wiese, Nucl. Phys. B356 (1991) 629;

A. S. Kronfeld and U.-J. Wiese, Nucl. Phys. B357 (1991) 521;

U.-J. Wiese, Nucl. Phys. B (Proc. Suppl.) 20 (1991) 24

[16] U.-J. Wiese, Nucl. Phys. B375 (1992) 45

[17] E. Brezin and J. Zinn-Justin, Nucl. Phys. B257 [FS14] (1985) 867;

V. Privman and M. E. Fisher, J. Stat. Phys. 33 (1983) 385

[18] C. Borgs and J. Z. Imbrie, Commun. Math. Phys. 145 (1992) 235

[19] C. Borgs, Berlin preprint, FUB-HEP 4/92, 1992, to appear in Nucl. Phys. B

[20] J.-D. Wang and C. DeTar, Salt Lake City preprint

[21] J. Bricmont, A. El Mellouki, and J. Fröhlich, J. Stat. Phys. 42 (1986) 743;

D. B. Abraham,"Surface Structures and Phase Transitions-Exact Results", in: "Phase Transitions and Critical Phenomena", vol. 11, ed. C. Domb, J. L. Lebowitz, Academic Press, London 1987;

K. K. Mon, D. P. Landau, and D. Stauffer, Phys. Rev. B42 (1990) 545;

V. Privman, in: "Workshop on the Dynamics of First Order Phase Transitions", HLRZ, June 1-3, 1992, ed. H. J. Herrmann and F. Karsch, World Scientific, Singapore 1992 (in press)

[22] M. Hamermesh, Group Theory and its Applications to Physical Problems, AddisonWesley, Reading, Massachusetts 1962

[23] N. A. Alves, B. A. Berg and S. Sanielevici, Nucl. Phys. B376 (1992) 218

[24] B. A. Berg and T. Neuhaus, Phys. Lett. B267 (1991) 249

[25] B. Grossmann and M. L. Laursen, in: "Workshop on the Dynamics of First Order Phase Transitions", HLRZ, June 1-3, 1992, ed. H. J. Herrmann and F. Karsch, World Scientific, Singapore 1992 (in press);

B. Grossmann, M. L. Laursen, T. Trappenberg and U.-J. Wiese, HLRZ preprint, HLRZ 92-31, 1992 